\title{
Videothoracoscopic treatment of oesophageal leiomyoma
}

\author{
G C Roviaro, M Maciocco, F Varoli, C Rebuffat, C Vergani, A Scarduelli
}

\begin{abstract}
Background-Oesophageal leiomyomas are usually so easily removed that thoracotomy seems out of proportion and thoracoscopic removal is therefore highly desirable.

Methods-Out of a total of 1003 thoracoscopic operations undertaken between July 1991 and December 1996, seven patients underwent thoracoscopic removal of oesophageal leiomyoma. All of them had been preoperatively studied by oesophagogastroscopy and computed tomographic scanning of the chest which had confirmed the presence of a lesion with benign features. The surgical technique required intubation with a double lumen tube. Operative access was gained through the right chest via three ports and a small utility thoracotomy in the inframammary sulcus. The mean operating time was 120 minutes.
\end{abstract}

Results-Conversion to open thoracotomy was necessary in one case with a very large horseshoe-shaped leiomyoma which was firmly adherent. The mean postoperative hospital stay was seven days. No intraoperative deaths or postoperative complications occurred.

Conclusions-The simplicity and safety of the thoracoscopic approach, combined with reduced surgical trauma and postoperative pain and functional and cosmetic advantages, make this technique the approach of choice for the removal of oesophageal leiomyomas.

(Thorax 1998;53:190-192)

Keywords: thoracoscopy; oesophageal leiomyoma; benign oesophageal tumours

Minimally invasive surgery, commonly employed in the treatment of numerous abdominal diseases, is finding an increasing field of application in thoracic surgery. ${ }^{12}$ Benign diseases are usually considered as elective indications for thoracoscopy because of the nature of the lesion and the relative simplicity of its removal for which a conventional thoracotomy would seem out of proportion. ${ }^{3}$ Benign oesophageal neoplasms, although rare, can be included in this group; leiomyoma is the most frequent and accounts for $70-80 \%$ of all benign oesophageal lesions. ${ }^{45}$ Most leiomyomas are localised in the middle or lower third of the oesophagus as single, smooth and well encapsulated submucosal masses. Mostly asymptomatic, they can cause dysphagia due to lumen encroachment. Removal is indicated not only when symptoms are present, but also before this stage is reached to prevent excessive growth which would complicate the operation. ${ }^{6}$ Considering the relative ease with which the lesion can be enucleated, thoracoscopy could represent the access of choice.

\section{Methods}

Of 1003 thoracoscopic operations performed from July 1991 to December 1996, seven patients (five men) of mean age 47 years (range 18-75) underwent enucleation of an oesophageal leiomyoma. Two patients were asymptomatic, the lesion having been discovered accidentally in one case during a follow up for gastric ulcer, and when admitted for physiotherapy and rehabilitation following an episode of cerebral ischaemia in the other case. Five patients presented with dysphagia and abdominal pain. All patients were subjected to upper digestive endoscopy. A narrow oesophageal lumen was found in all cases due to extrinsic compression by the tumour and intact overlying mucosa. The tumour was localised in six cases in the middle third and in one case in the lower oesophagus. Reflux oesophagitis was present in two patients. A transparietal biopsy specimen was obtained in one patient as the mass was particularly large. Computed tomographic (CT) scanning of the chest confirmed the benign nature and features of the lesions which were characterised by uniform density and no sign of infiltration of nearby structures nor of mediastinal lymphadenopathy. All patients had a single lesion ranging from $2 \mathrm{~cm}$ to $7 \mathrm{~cm}$ in length. In one case CT scanning demonstrated a mass involving two thirds of the oesophageal circumference.

\section{OPERATIVE TECHNIQUE}

After induction of general anaesthesia using a double lumen endobronchial tube, the patient was placed in the left lateral decubitus position and the right lung was excluded by selective ventilation. Once collapse had been achieved, two $10 \mathrm{~mm}$ trocars were inserted into the fifth intercostal space on the anterior and posterior axillary line. Another trocar for the optic was inserted in the sixth or seventh intercostal space on the mid axillary line, according to the site of the lesion. A small utility thoracotomy of 4-5 centimetres in length was created in the inframammary sulcus in order to extract the tumour and to insert non-endoscopic instruments, should the need arise (fig 1). The operating table was tilted slightly towards the left side in order to displace the lung anteriorly and to facilitate access to the posterior mediastinum and exploration of the thoracic oesopha- 


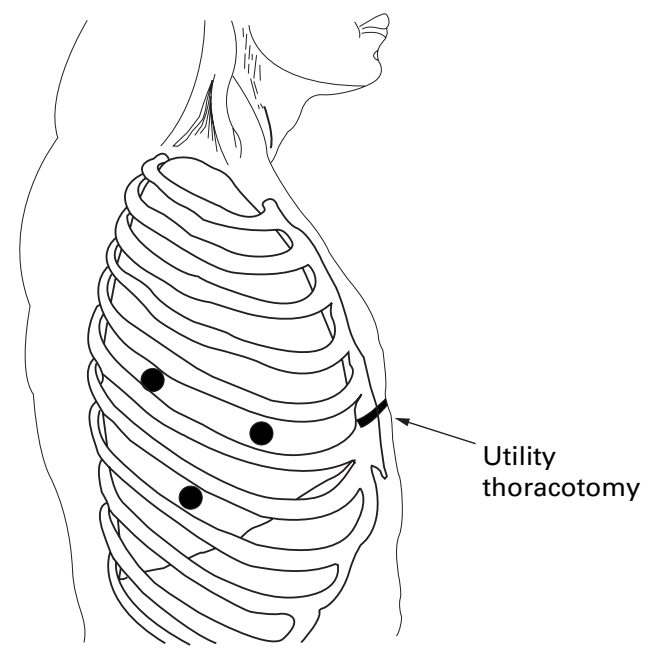

Figure 1 Site of the trocars and utility thoracotomy. Two $10 \mathrm{~mm}$ trocars are inserted in the fifth intercostal space on the anterior and posterior axillary line. Another trocar for the optics is inserted in the sixth or seventh intercostal space on the mid axillary line. A utility thoracotomy a few

centimetres long is carried out in the inframammary sulcus.

gus. The mediastinal pleura was incised over the lesion, which appears as an alteration of the oesophageal profile. When it cannot be seen, the tumour can usually be detected within the oesophageal wall using a mounted swab. When the lesion is located in the middle third of the oesophagus it can be identified more easily after dividing the arch of the azygos vein with an endoscopic stapler. Once the parietal pleura had been opened, the longitudinal muscular fibres of the oesophagus were incised and the lesion was then enucleated by careful dissection using a mounted swab (fig 2). The tumour can be manoeuvred more easily if it is clipped or transfixed with a stitch. When the tumour is particularly adherent to the mucosa, transillumination by intraoperative oesophagoscopy can facilitate isolation, dissection, and enucleation. The leiomyoma was then inserted into a bag and extracted through a port site or

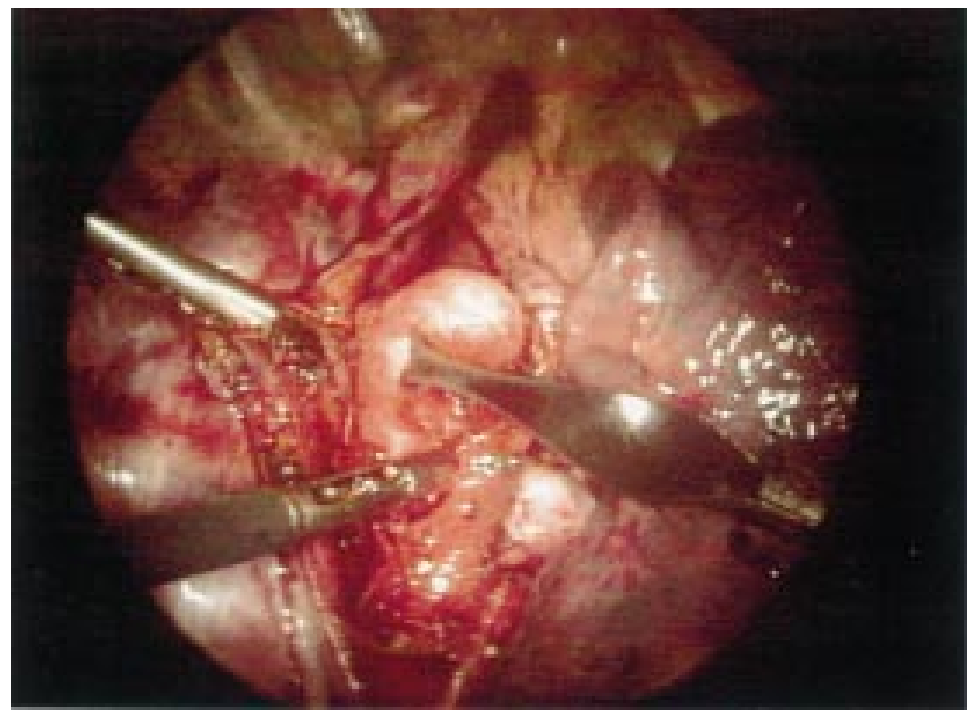

Figure 2 Once the lung is displaced anteriorly the azygos arch is divided and the mediastinal pleura is opened. The longitudinal fibres of the oesophagus are spread apart and the leiomyoma is enucleated with the aid of a mounted swab. through the utility thoracotomy, depending on its size.

\section{Results}

The mean operating time was 120 minutes (range 90-150). Histological examination confirmed the diagnosis of leiomyoma, the diameter of the lesion varying from 2.5 to $9 \times 4 \mathrm{~cm}$. There was no intraoperative mortality and no postoperative complications were recorded. The mean postoperative hospital stay was seven days (range 6-10).

Only in one case was conversion to open thoracotomy necessary. The patient had a particularly large horseshoe-shaped mass on the lower oesophageal segment and had undergone preoperative transparietal biopsy. Conversion was unavoidable in order to enucleate the mass which was very firmly adherent to the oesophageal mucosa, as well as to repair an accidental lesion of the mucosa that had occurred while trying to isolate the tumour. The postoperative course was uneventful and the patient was discharged on the seventh postoperative day. None of the lesions have recurred during a mean follow up of 37 months (range 10-65 months).

\section{Discussion}

Even though only recently introduced, the advent of thoracoscopic surgery has already become accepted as the treatment of choice for many thoracic diseases such as bullectomy, pleurectomy for spontaneous pneumothorax, or wedge resection in the case of peripheral metastasis or primary lung cancer. ${ }^{1-11}$ Oesophageal surgery is also indicated in the field of minimally invasive techniques and malignant oesophageal tumours are removed thoracoscopically in a few centres, but the oncological validity of this is still controversial. ${ }^{3}$ In the case of benign tumours such as a leiomyoma, however, positive results of the videothoracoscopic approach are indisputable..$^{3-5}$ The advantages of thoracoscopic removal of such lesions are confirmed in the limited series that have been published in recent years; particularly significant is the relative simplicity of this kind of approach compared with traditional thoracotomy. ${ }^{3-5}$

Leiomyomas usually grow within the oesophageal wall. The tumour is usually asymptomatic and may be discovered incidentally in the course of examinations carried out for other reasons. The characteristics of the lesion are clearly seen using oesophagoscopy and conventional imaging techniques (barium swallow, CT scanning, endoscopic ultrasound). ${ }^{12}$ Endoscopic biopsy specimens are unnecessary and may even be contraindicated as inflammatory adhesions may develop which alter the cleavage planes and induce the risk of injury to the mucosa while attempting to shell out the tumour. In the only case which we were compelled to convert to thoracotomy, the lesion had been defined as a voluminous posterior mediastinal "neoplasm"; biopsy specimens were non-diagnostic and the histology was assessed at frozen section during thoracoscopy. 
Not only the size of the mass but probably also the transoesophageal biopsy contributed to the mucosal lesion.

Most authors agree that the tumour should be removed even in asymptomatic patients, not only to assess the diagnosis but also to remove the mass before its growth, even though slow, hinders removal with a totally unjustified risk of complications. The operation is technically simple whether carried out as a thoracotomy or a thoracoscopy. However, routine manoeuvres can become quite troublesome with thoracoscopy because of the lack of manual palpatory perception and the inadequacy of endoscopic instrumentation. This is particularly true in the case of horseshoe-shaped leiomyomas as the muscle fibres tend to become fragmented and lacerated with endoscopic instrumentation. This can result in complications when suturing the myotomy.

Should the myotomy be sutured after having enucleated the tumour if the mucosa is intact? Reports in the literature are not in agreement. Most agree on the necessity of approaching the muscle fibres. According to Hennessy and Cuschieri, a wide breach with widely separated fibres "can be left without causing concern, provided the mucosa is intact". ${ }^{13}$ On the other hand, Peracchia states that suturing the breach is essential in order to prevent mucosal bulging. ${ }^{34} \mathrm{He}$ also reports that at the beginning of his experience he had opted for not suturing the muscular layer because of insufficient skill in minimally invasive suturing techniques. The patient had afterwards complained of dysphagia. At manometry an aperistaltic tract was discovered; the patient was therefore reoperated and the muscular layer was sutured, successfully resolving both symptoms and manometric abnormalities. We agree with the general principle of suturing the muscle fibres and have done so using Endohernia clips in one case and interrupted 3/0 Vicryl stitches in four other cases. However, in two cases the muscle was not sutured because of technical difficulties or excessive fragmentation of the muscle fibres and the postoperative course and follow up in these patients was totally normal. It is essential to be absolutely sure that the mucosa is intact as an unrecognised mucosal lesion can produce important postoperative complications. Intraoperative endoscopy is invaluable in allowing the surgeon to check the integrity of the mucosa during all steps of the enucleation procedure. The presence of the endoscope also provides transillumination which improves visualisation of the operative field and stabilises the oesophagus. ${ }^{14}$

A thoracoscopic approach offers potential advantages compared with traditional thoracotomy including improved functional and aesthetic disability, reduced pain, and improved respiratory function. The limited operative trauma should allow a reduced postoperative hospital stay and more rapid resumption of normal activity. The relatively prolonged postoperative hospital stay in our patients is due to several factors. We have adopted a conservative approach to postoperative management including a Gastrografin swallow on the fifth postoperative day. Also our patients often come from a considerable distance from the hospital and our public health system does not provide intermediate assistance.

1 Roviaro GC, Rebuffat C, Varoli F, et al. Videoendoscopic thoracic surgery. Int Surg 1993;78:4-9.

2 Mack MJ, Aronoff RJ, Acuff TE, et al. Present role of thoracoscopy in the diagnosis and treatment of diseases of the coscopy in the diagnosis and treatment
chest. Ann Thorac Surg 1992;54:403-9.

3 Peracchia A, Ancona E, Ruol A, et al. Minimally invasive techniques in esophageal surgery. In: Steichen FM, Welter $\mathrm{R}$, eds. Minimally invasive surgery and new technology surgery. St Louis: Quality Medical Publishing, 1994: 538-41.

4 Peracchia A, Rosati R, Bardini R. Thoracoscopic removal of benign tumors of the esophagus. In: Toouli J, Gossot D, Hunter JG, eds. Endosurgery. Edinburgh: Churchill Livingstone, 1996: 267-73.

5 Gossot D, Fourquier P, El Mteini M, et al. Technical aspects of endoscopic removal of benign tumors of the esophagus. Surg Endosc 1993;7:102-3.

6 Roviaro GC, Rebuffat C, Varoli F, et al. Videothoracoscopic excision of mediastinal masses. Ann Thorac Surg 1994;58: 1679-84.

7 Miller DL, Allen MS, Trastek UF, et al. Videothoracoscopic wedge excision of the lung. Ann Thorac Surg 1992;54:4104.

8 Varoli F, Roviaro GC, Rebuffat C, et al. Traitment video-thoracoscopique du pneumothorax spontaneé. $\mathcal{F}$ Celio-Chirurg 1996;17:27.

9 Roviaro GC, Varoli F, Rebuffat C, et al. Thoracoscopic pulmonary resection. Ann Thorac Surg 1995;60:1155-62.

10 Roviaro GC, Varoli F, Rebuffat C, et al. Videothoracoscopic staging and treatment of lung cancer. Ann Thorac Surg

11 Varoli F, Roviaro GC. Technique of thoracoscopic retrieval of the lung. Min Invas Ther Allied Technol 1996;5:147-9.

12 Boyce GA, Sivak MV Jr, Rosch T. Evaluation of submucosal upper gastrointestinal lesions by endoscopic ultrasound. Gastrointest Endosc 1991;37:449-54.

13 Hennessy TPJ, Cuschieri A. Tumours of the oesophagus. In: Hennessy TPJ, Cuschieri A, eds. Surgery of the oesophagus. London: Butterworth-Heinemann 1992: 275-327.

14 Peracchia A, Segalin A, Bonavina L, et al. Intraoperative endoscopy for minimally-invasive esophageal surgery. Endosurgery 1994;2:53-5. 